\title{
Details behind the dots: How different intensive care units used common and contrasting methods to prevent ventilator associated pneumonia
}

\author{
Malcolm Daniel, Malcolm Booth, Kirsteen Ellis, Shaun Maher, Andrew Longmate \\ Forth Valley Royal Hospital and Glasgow Royal Infirmary
}

\begin{abstract}
Care bundles promote delivery of effective care and improve patient outcomes. The understanding of how to improve delivery of care bundles is incomplete.

The Scottish Patient Safety Programme is a national collaborative with the aim of improving the delivery of care to patients in acute hospitals in Scotland. Critical care is one of five workstreams in the programme. A programme goal is to reduce incidence of ventilator-associated pneumonia (VAP) to zero or 300 calendar days between events through use of a VAP Prevention bundle. We studied two ICUs participating in this programme. Each ICU had established infection surveillance system prior to the programme starting. Both units had an appreciable incidence of VAP. Initial VAP prevention bundle adherence was low in each ICU (35\% and $41 \%$ ).
\end{abstract}

Comparing time periods before and after $80 \%$ bundle VAP prevention bundle adherence was achieved showed a similar reduction in VAP incidence (from 6.9 to 1.0 , and from 7.8 to $1.4 / 1000$ ventilation days). When compared each ICU used common and contrasting approaches to accomplish this improvement.

We describe the five improvement knowledge systems used to improve bundle adherence to bundle elements in each hospital. The insights gained from these front-line clinical teams can be used as a template for improvement efforts in a variety of other healthcare settings.

\section{Problem}

Care bundles (defined sets of clinical actions) promote a systems approach to improving delivery of effective treatments. Measuring adherence to the care bundle as "all-or-none" encourages the design of a whole package of care rather than individual parts. This maximizes the clinical benefit to be gained from use of a care bundle.[1]

Ventilator-associated pneumonia (VAP) is the most common nosocomial ICU-acquired infection and has important associated morbidity, mortality and costs.[2,3] Ventilator bundles and ventilatorassociated pneumonia (VAP) prevention bundles have been used in a number of intensive care units (ICUs) and are associated with reductions in VAP incidence.[4-8] An Institute for Healthcare Improvement $(\mathrm{IHI})$ collaborative demonstrated that ICUs achieving high ventilator care bundle adherence achieved the greatest reductions in VAP.[9] This report did not describe how units achieving high adherence rates had accomplished this.

This characterizes the widespread gap that exists between knowledge of which treatments are effective and knowledge on how to improve delivery of those treatments in the front-line setting. To date, detailed descriptions of behaviour change interventions to improve bundle adherence are limited. The need for improved understanding and validation of such improvement work has been identified.[10-12]

This report describes the implementation of a VAP prevention bundle in ICUs in two separate hospitals where each unit started with low levels of bundle adherence and appreciable incidences of VAP. We detail the common and contrasting approaches to improving bundle adherence, and offer the learning gained from our reflection on this work for application to other areas of clinical practice.

\section{Background}

The Scottish Patient Safety Programme [13] was launched in January 2008. The programme aims are to improve the safety of health care in adult hospitals across Scotland. The Institute for Healthcare Improvement was the technical partner for this work. The Scottish Patient Safety Programme used a Breakthrough Series Collaborative model [14], this included five national learning sessions in the first two years. There are five workstreams involved, one of which is critical care. One of the programme goals was to improving bundle adherence and achieve zero incidence of VAP rate or 300 calendar days between episodes by 2012 .

At the start of SPSP the Scottish Intensive Care Society (SICS) published an evidence based VAP prevention bundle.[15] This was a strategic decision for the Scottish Patient Safety Programme to build on work already undertaken by the evidence-based medicine group of the SICS. The theory behind this approach was using a bundle developed by leading Scottish clincians would improve clincian engagement with the work.

We used a time series study design [16] and report VAP prevention 
bundle adherence and VAP rates in the ICUs of two hospitals 25 miles apart. We evaluated the mechanisms of behaviour change $[17,18]$ between October 2005 and April 2010. Local research ethical committee review was not required [16] and the local research ethics committee confirmed this.

Stirling Royal Infirmary (NHS Forth Valley) ICU has nine beds and approximately 400 admissions per year. Glasgow Royal Infirmary ICU has nine beds and approximately 425 admissions per year. Each unit is the only adult ICU in the hospital, takes medical and surgical patients, uses a closed model of care, and had a stable workload over the study period.

In both ICUs adherence to the VAP prevention bundle was measured on an all-or-none basis.[1] All four elements had to be performed for the bundle to be judged as delivered. The four bundle elements were:

1. Sedation to be reviewed and, if appropriate, stopped on a daily basis to allow titration of effect

2. All patients will be assessed for weaning and a daily weaning plan identified and commenced

3. Avoid the supine position, aim to nurse the patient at least $30^{\circ}$ head up

4. Use chlorhexidine antisepsis as part of daily mouth care.

\section{Baseline measurement}

Bundle adherence was measured daily in the Stirling ICU by charge nurse review of each patient's charts. In the Glasgow ICU each patient had an ICU daily goals sheet recorded. These were collected on a weekly basis. Bundle adherence was measured by sampling the daily goals sheet from one day in each week. The day was selected by use of a random number generator. In each ICU monthly bundle adherence was calculated from the data gathered and plotted on a run chart.

At Stirling the run chart was displayed on the wall in the charge nurse office (within the ICU). This was updated monthly. The run chart was shown at weekly lunchtime meetings that included staff working on the unit on that day. In the Glasgow ICU the run chart was placed on a designated area in an L-shaped corridor that the staff used to get to the coffee room. The run chart was in direct line of sight as staff walked down the first part of the corridor. The ICU doctor also used brief weekly huddles at the run chart to discuss progress with the bundle with nursing and medical staff. This started in May 2008 and focussed on a run chart with VAP bundle adherence plotted on one the primary $y$-axis, and VAP rate on the secondary $y$-axis. This helped engage staff in the work as they saw the linkage between improving a VAP bundle adherence and reducing the incidence of VAP. Details and exact criteria for recording bundle adherence in each unit are shown in table 1.

Initial bundle adherence was $41 \%$ in the Stirling ICU and $35 \%$ in the Glasgow ICU.

Each ICU had previously participated in hospital acquired infection surveillance.[19] Each patient admitted to the ICU for greater than
48 hours was screened daily for hospital acquired infection. The method for this was review of the 24 hour observation chart, medical records, laboratory results, and chest $x$ ray. A simple diagnostic flowchart was used (at SRI) to standardise decision making (figure 1). Nurses and doctors caring for patients (who were often not directly involved in leading the improvement work) were questioned about the data fields. The individual elements collected by the surveillance team and analysed using objective criteria to diagnose VAP as defined by the HELICS (Hospitals In Europe Link for Infection Control through Surveillance) criteria (figure 1).[20]

A diagnosis of VAP was further classified by microbiological diagnostic method into HELICS categories PN1-6. Neither ICU used bronchial alveolar lavage to diagnose VAP. PN 4 was the most common diagnostic category in both ICUs $(>90 \%)$. The definition of this category is based on positive sputum culture or nonquantitative lower respiratory tract specimen culture. The diagnostic definition remained constant during this study.

Chest $\mathrm{x}$ rays were performed routinely on admission to both ICUs or following tracheal intubation, central venous catheter insertion, tracheostomy and as clinically indicated. Interpretation of chest $\mathrm{x}$ rays was performed by use of formal radiology reports or direct consultation with senior medical staff on the unit. Routine endotracheal aspirate samples were taken on admission; on Monday, Wednesday, and Fridays. Additional surveillance samples were taken as clinically indicated. Each ICU had daily communication with a microbiologist to assess and interpret microbiology results. Patients were not followed after discharge from ICU.

Crosschecking all names against the ICU admission book and the ICU electronic database made confirmation that every patient was captured. The method of data collection has been validated nationally in Scottish ICUs by independent reviewers, the method has a sensitivity of $74.5 \%$ and specificity of $94.5 \%$ for detecting VAP.[21, 22]

Surveillance has run continuously from September 2005 using trained nurses in the Stirling ICU. In the Glasgow ICU, a trained doctor had done this intermittently from 2004, and continuously from October 2007. In each ICU this pre-dates the start of the SPSP.

Incidence of VAP per 100 ventilator days was displayed on a monthly basis along with VAP prevention bundle adherence. A ventilator day was defined as a patient receiving positive pressure ventilation via endotracheal tube or tracheostomy tube for any part of the day.

The run chart of monthly VAP prevention bundle adherence and VAP rate per 1000 ventilated days in each unit are shown in figures 2 and 3.

See supplementary file: ds4644.pptx - "Figure 1, Table 1, Figure 2, Figure 3"

\section{Design}


A few members of each ICU team attended the five national learning sessions in the first two years. Each learning session included education on the model for improvement,[23] this was the improvement methodology used as part of the national programme.

The Stirling ICU had previously developed and provided staff education on quality improvement. This began with regular critical care improvement group meetings. This occurred when the ICU was created a few years earlier from the amalgamation of two separate ICUs. The ICU employed a full time nurse educator to work on improving and standardising care in a number of areas including the care of ventilated patients, initiation of early enteral feeding, introduction of renal replacement therapy capability, and a standardised approach to sedation. There had been efforts to develop an atmosphere of collaboration between medical and nursing staff. The Stirling ICU also had previous experience using a bundle program to prevent VAP [24] this included education of staff and intermittent measurement of bundle adherence.

The Glasgow ICU had not previously attempted process or outcome improvement. In both hospitals parallel interventions were being implemented with the aim of reducing central venous catheter related bloodstream infections during the period of the study.[25]

\section{Strategy}

Two of the four elements were responsible for the majority of nonadherence to the bundle in both ICUs. These were: 1) failure to stop sedation on a daily basis to allow titration of effect, and 2) failure to identify and commence weaning from the ventilator. They accounted for $83 \%$ and $93 \%$ of the bundle non-adherence in the Glasgow and Stirling ICU respectively.

Methods for increasing bundle adherence

Glasgow Royal Infirmary: Reasons for low adherence with weaning ventilation included lack of clarity of daily goals, of which the VAP bundle was a part. A daily goals sheet had previously been developed to set goals and record bundle adherence. This was another part of the SPSP critical care workstream. There was a dedicated free text space labelled "Weaning plan for next 24h" rather than a tick-box. The text entered on the ward round often stated when to stop weaning, rather than a weaning target to work towards. The space for recording the weaning plan was modified to include prompts for recording specific ventilator settings. We found the daily goals were often written but not communicated. This led to lack of clarity about the goals other than for the individual who had written them. A script of questions for the bedside nurse to ask medical staff on the morning ward round was tested and developed. These questions clarified the target level of ventilator support to be worked towards that day. Elements defined in this target included ventilator mode, pressure support level (pressure support is the mode of ventilation most commonly used in this ICU), level of positive end expiratory pressure, and inspired oxygen concentration. The process of asking and answering these questions clarified the goal for each patient among all members of the team on the ward round. The nurses reported this made their daily work much clearer. An assessment of progress with the weaning plan was performed on the afternoon ward round.

Reasons for low adherence to sedation break discovered in interviews with staff included lack of clarity on what to do if sedation needed to be restarted; this ambiguity made staff reluctant to perform the sedation break. A departmental "protocol" for sedation break had been written in February 2008. It had been written on a sheet of paper and included details of the study results of this intervention. A copy of the protocol was placed in a folder at each bed space. The protocol was too detailed and was not read or used by staff. This was redrafted, tested, and simplified to 15 words: "Stop. If need to start again - start at $1 / 2$ previous rate and titrate to effect." The timing of doing stopping the sedation was decided on the ward round, taking into account other care processes going on for that patient (for example trips to radiology). The simplified message clarified what to do if the sedation needed to be restarted and removed a barrier to the sedation breaks happening. The simplified message was integrated into the daily goals chart as a prompt, and onto the script of questions for the nurses.

Stirling Royal Infirmary: The reasons for low adherence were explored by discussions with staff and assessment of behaviour patterns. These were noted when the charge nurse performed the daily checks. It was clear which staff had not performed bundle elements. The pattern of this was evaluated over several days and weeks. Individuals who consistently did not deliver care including the bundle elements after standard education and simple verbal requests were identified and engaged in more detailed conversation and appreciative inquiry. Reasons for low adherence with the policy on sedation break included: not fully understanding the benefits of the break for patients; feeling that it might be cruel; with specific concerns about pain relief and stopping analgesic infusions, concerns about situations when it might cause harm (risk of accidental extubation, or in patients with a head injury, or those receiving palliative care) and a feeling of "loss of control." Nursing staff did not understand that they were allowed to recommence sedative and analgesic infusion as required after a period of cessation.

In response to this information, staff were educated about the benefits of sedation break, and the importance of being expected to recommence sedation after a break, if required, was emphasised. Informal and formal discussions were had - informal at the bedside and formal at weekly nursing SPSP meeting on the ward (in the coffee room). Open discussion was encouraged and attempts made to better understand the anxieties and beliefs motivating behaviour. Particular nurses who were reluctant to deliver bundle elements were identified by their behaviours (this was apparent when the charge nurse performed the daily check). Three senior (older) nonadherent nurses who were key opinion influencers on the nursing staff were identified. These individuals were often in charge of shift and therefore powerfully influenced other nurses' behaviour and the expectations. These individuals were approached by the charge nurses for one on one meetings to discuss the rationale for the sedation break, exchange views and explicitly asked for their support and change in behaviour.

We noticed that some nurses performed weaning from ventilation 


\section{BMJ Quality Improvement Reports}

(reduction in pressure support level, reduction in fraction inspired oxygen level and adjustments to positive end expiratory pressure) autonomously while many others were not confident to without this being requested to by a doctor. When we spoke to those nurses who did not reduce ventilator support the reasons identified were concerns that medical staff would not be supportive of this and that they were not confident to do this safely without causing harm for the patients. There was also difficulty understanding the existing unit weaning guidelines. Medical support for nurse led ventilator weaning was reaffirmed at ICU management meetings and by behaviours on the ward. Using small tests of change a team that included a consultant doctor and nurse, neither of who was involved with original development of the VAP bundle, reassessed and improved the existing weaning guidelines into a simpler flow chart. Less experienced nurses found the flowchart useful in empowering and guiding reductions in ventilator pressure support. A verbal prompt was encouraged and used on the daily ward round by asking the nurse at the bedspace, "Where are you on the weaning flow chart?" This proved to be a useful way to ensure understanding and support practice.

A self-adhesive reminder label for ventilator weaning and sedation break was introduced on the nurse's observation chart. This label which included a written prompt requiring a yes-or-no answer to the statement "Reduce support using ventilation management flow chart," and a space to record the outcome of any weaning trial was applied to the notes every day. In addition, a sedation stop and weaning plan prompt were added to the charge nurse's daily checklist as a "tick box" and completed daily for every patient. When a patient developed a VAP the charge nurse carried out a root cause analysis. All elements of bundle delivery were checked for the duration of the patient's stay. Any episodes of incomplete adherence were communicated to and discussed with staff. This was done at lunchtime meetings with nursing staff. Information was received positively; this appeared to lead to a greater understanding of importance of bundle elements. Patient stories: patients whom developed VAP had their case history evaluated and a description of their complication presented at regular nursing meetings. There was a weekly lunchtime nurse SPSP meeting when these instances where discussed.

\section{Results}

VAP prevention bundle adherence and VAP rate

The run chart of monthly VAP prevention bundle adherence and VAP rate per 1000 ventilated days in each unit are shown in figures 2 and 3 . The pattern of process improvement and change in outcome are similar between each ICU.

VAP incidence rate ratios were compared between the time periods before and after $80 \%$ bundle adherence was achieved by calculating the incidence rate ratio and $95 \%$ confidence interval using Stata v10.0.

The level of $80 \%$ all-element reliability was both challenging and potentially achievable as a pragmatic initial systems goal, in light of previous documented level of 54\% adherence.[24] Table 2 compares VAP incidence rate before and after $80 \%$ bundle reliability was achieved.

See supplementary file: ds4744.pptx - "Table 2 Figure 2 Figure 3"

\section{Lessons and limitations}

The experience described in this paper validates previous improvement work,[10-12] confirming the association between achieving high VAP prevention bundle adherence reducing VAP incidence.[4-8] It also contributes to the development of hypotheses on mechanisms of change in human performance [26] by describing in detail the common and contrasting approaches used at the front line of clinical practice in each ICU to achieve this.

The origin of this report began when two doctors (AGL and MD) noticed the similar pattern of improvement in run charts collected by each ICU. Through conversations they had about how each ICU had improved came the realization that although process improvement in both units was based on principles that were held in common, there were also marked differences in execution between each ICU.

Five knowledge systems have been identified to support improvement,[27] including 1) generalizable scientific evidence; 2) awareness of the particular context; 3) development and application of systems for valid, reliable measurement of outcomes; 4) plans for change that adapt generalizable evidence to the local context; and 5) execution of planned changes. This report addresses these knowledge systems in the following way. Table 4 shows how the first four knowledge systems were addressed in the improvements described. The approaches used had a great deal in common.

1. Generalisable scientific evidence provided the basis of the VAP prevention bundle. An existing network of clinicians interested in infection control and the application of evidence-based medicine developed the Scottish Patient Safety Programme's VAP prevention bundle. One clinician from each ICU had been involved in the development of this work (AGL and MGB). The bundle used was not only based on evidence but also had an element of very local ownership in each ICU

2. The particular local contexts were broadly similar in each ICU; they are in the same country. Surveillance of infection was not standard practice in Scottish ICUs prior to SPSP. Only six of $29 \mathrm{ICUs}$ had ever measured hospital acquired infection surveillance data. This was for a six month nonsustained pilot period.[21, 22] Each ICU in this study had participated. Each ICU had existing experience and expertise in collecting such outcome data. Only the Stirling ICU had measured reliability of processes of care. This was intermittent and was not sustained despite sub-optimal performance. The Stirling ICU had established structures represented by meetings to provide education for staff on quality improvement. Education was an important method for the Stirling ICU, and this helped shape its approach to SPSP. The Glasgow ICU did not have this previous experience, the work here focussed on execution of 
changes at the front-line

3. Performance measurement was by analysis of time series data in both ICUs. Similar improvements were demonstrated over time. Submission of monthly data was required for SPSP. The ICUs measured bundle adherence at different frequencies, daily versus a weekly sample. This did not appear to affect progress. Both ICUs displayed data visually. The Glasgow ICU plotted both bundle and outcome on the same run chart, and found the linkage between improving a process and improving an outcome a powerful tool for engaging staff in the work. Both ICUs made efforts to ensure that clinical staff understood and were aware of process and outcome measures

4. The plans for change shared many common elements. Initiation of the Scottish Patient Safety Programme was a strong external influence and provided a common improvement methodology and aims. Each ICU made many sequential changes, the learning from what had worked and what had not led to further changes. As noted above, the weighting towards education of staff or execution of changes varied between the ICUs

5. The knowledge of how to make things happen is key to execution of planned changes. In each ICU senior experienced members of the clinical team led the improvement work. This gave prominence and attention to the work. The Stirling ICU had some key opinion leaders, who were at the laggard end of the diffusion of innovation curve. This provided a barrier to progress. The local leaders held these key individuals accountable for their actions. This was an important barrier to overcome. The Stirling ICU had previously been unable to sustain process measurement by the ICU nurses. The Board level support for SPSP resulted in a transactional approach of aligning leadership priorities with the work of the local team that enabled this to happen. Both ICUs celebrated of success, marked by each time the ICU achieved a further 100 calendar days following the last episode of VAP. This open recognition of an "early-win" helped keep staff engaged.

Limitations: The findings of this paper need interpretation in the light of some limitations. Firstly, the work undertaken was part of a national programme on patient safety. The aims of the programme provided an incentive for each team to design a highly reliable process and be successful. The existence of this program may be considered to limit the generalisability of our findings to other healthcare environments without similar programs. This does, however, emphasize the importance of leadership involvement, aim setting, and a focus on systems in all improvement projects.[28] Secondly, we have described our local contexts at this point in time; there may have been earlier experiences that have influenced our ability to do this work that we did not recognize. Thirdly, each ICU's previous voluntary participation in reporting infection outcomes does display a commitment to quality. It was only when the ICUs were forced, by involvement in SPSP, to look at underlying processes that improvement in clinical outcomes happened. Fourthly, the work has been described on the basis of five previously described knowledge systems.[27] It is likely there was interaction between these knowledge systems that we have not been able to describe. Fifthly, there may be concern regarding the rigor of this study.

This paper demonstrates that reflecting on experiential learning gained is an integral part of all improvement work. This includes comparing and learning from our colleagues working in different hospitals.

\section{Conclusion}

Changing front-line clinical practice is challenging and complex work. Quality improvement is a social discipline [29] that is influenced by the particular local context. Context has been described as everything that is not the intervention itself.[30] In responding to the call to describe the context of interventions [31] we have reviewed, reflected, and shared how each ICU made changes happen.

While a run chart may show impressive improvement, it is the "details behind the dots" that allows generation of hypotheses on how these changes were achieved.

We have taken a simple formula [27] to describe how different forces in each unit combined to produce the improvement in clinical outcomes.

Similar improvement was accomplished in each ICU. Education was a stronger theme in one ICU, while the other focused on execution of changes at the front-line. The learning gained from this work has updated the approach used in each ICU. The Glasgow ICU has developed weekly meetings to educate staff and shape current improvement work. The Stirling ICU has increased efforts to ensure process execution at the bedside. We believe our experience and learning will help others wanting to improve healthcare delivery in different settings.

\section{References}

1. Nolan T, Berwick DM. All-or-none measurement raises the bar on performance. JAMA 2006;295:1168-70.

2. Vincent JL, Bihari DJ, Suter PM, Bruining HA, White J, Nicolas-Chanoin $\mathrm{MH}$, et al. The prevalence of nosocomial infection in intensive care units in Europe. Results of the European Prevalence of Infection in Intensive Care (EPIC) Study. EPIC International Advisory Committee. JAMA 1995;274:639-44.

3. Heyland DK CD, Griffith LE, Keenan SP, Brun-Buisson C for the Canadian Critical Care Trials Group. . The attributable morbidity and mortality of ventilator-associated pneumonia in the critically ill patient. Am J Resp Crit Care Med 1999;159:1249-56

4. Berenholtz SM, Pham JC, Thompson DA, Needham DM, Lubomski LH, Hyzy RC, et al. Collaborative cohort study of an intervention to reduce ventilator-associated pneumonia in the intensive care unit. Infect Control Hosp Epidemiol 2011;32:305-14

5. Al-Tawfiq JA, Abed MS. Decreasing ventilator-associated 
pneumonia in adult intensive care units using the Institute for Healthcare Improvement bundle. Am J Infect Control 2010;38:552-6.

6. Marra AR, Cal RGR, Silva CV, Caserta RA, Paes AT, Moura DF, Jr et al. Successful prevention of ventilatorassociated pneumonia in an intensive care setting. Am J Infect Control 2009;37:619-25.

7. Blamoun J, Alfakir M, Rella ME, Wojcik JM, Solis RA, Anees Khan M, et al. Efficacy of an expanded ventilator bundle for the reduction of ventilator-associated pneumonia in the medical intensive care unit. Am J Infect Control 2009;37:172-5

8. Bonello RS, Fletcher CE, Becker WK, Clutter KL, Arjes SL, Cook JJ, et al. An intensive care unit quality improvement collaborative in nine Department of Veterans Affairs hospitals: reducing ventilator-associated pneumonia and catheter-related bloodstream infection rates. Joint Comm J Qual Pat Saf 2008;34:639-45.

9. Resar R, Pronovost P, Haraden C, Simmonds T, Rainey T, Nolan $\mathrm{T}$. Using a bundle approach to improve ventilator care processes and reduce ventilator-associated pneumonia. Joint Comm J Qual Pat Saf 2005;31:243-8.

10. Ranji S, Shetty K, Posley K, Lewis R, Sundaram V, Galvin $\mathrm{C}$, et al. Prevention of Health Care Associated Infections. Rockville, MD: Agency for Healthcare Research and Quality.; 2007; Available from: www.ahrq.gov/downloads/pub/evidence/pdf/qualgap6/hainfg ap.pdf Accessed 7th January 2015.

11. Pronovost PJ, Berenholtz SM, Morlock LL. Is quality of care improving in the UK? BMJ 2011;342:c6646.

12. Bosk CL, Dixon-Woods M, Goeschel CA, Pronovost PJ. Reality check for checklists. Lancet 2009;374:444-5.

13. Scottish Patient Safety Programme Available from: http://www.scottishpatientsafetyprogramme.scot.nhs.uk/prog rammes/acute-adult Accessed 7th January 2015.

14. The Breakthrough Series: IHI's Collaborative Model for Achieving Breakthrough Improvement. Boston, MA: Institute for Healthcare Improvement; 2003.

15. Beard D BM, Cook B, Cole S, Crofts S, Curran S, Kellagher A, Longmate A, Swann D. VAP Prevention Bundle: Guidance for Implementation. . Scottish Intensive Care Society Audit Group. 2012; Available from: http://www.sicsag.scot.nhs.uk/HAI/VAP-Prevention-Bundleweb.pdf Accessed 7th January 2015.

16. Ogrinc G, Mooney SE, Estrada C, Foster T, Goldmann D, Hall LW, et al. The SQUIRE (Standards for QUality Improvement Reporting Excellence) guidelines for quality improvement reporting: explanation and elaboration. BMJ Qual Saf 2008;17:i13-i32.

17. Pawson R, Tilley N. Realistic Evaluation. Lodon, England: Sage Publications Ltd; 1997.

18. Berwick DM. The science of improvement. JAMA 2008;299:1182-4.

19. Cairns CJ, Reilly J, Booth M. Prevalence of healthcareassociated infection in Scottish intensive care units. $\mathrm{J}$ Hosp Infect. 2010;76:308-10.

20. Hospitals In Europe Link for Infection Control through Surveillance (HELICS). Surveillance of Nosocomial
Infections in Intensive Care Units (2004) Protocol Version 6.1.: HELICS 2004; Version 6.1: [Available from: http://www.ecdc.europa.eu/en/activities/surveillance/HAl/Do cuments/0409 IPSE ICU protocol.pdf Accessed 7th January 2015.

21. Scotland HP. Surveillance of Intensive Care Unit Associated Infections. Pilot report. Edinburgh, Scotland: Health Protection Scotland; 2005; Available from: http://www.hps.scot.nhs.uk/haiic/sshaip/publicationsdetail.as px?id=30291 Accessed 7th January 2015.

22. Scotland HP. Intensive Care Unit Associated Infection National Surveillance Programme Pilot Report - 2011. Glasgow, Scotland: NHS National Services Scotland; 2011; Available from: http://www.documents.hps.scot.nhs.uk/hai/sshaip/publicatio ns/icu-surveillance/icu-report-2011-03-30.pdf Accessed 7th January 2015.

23. Langley GJ, Moen R, Nolan KM, Nolan TW, Norman CL, Provost LP. Using the Model for Improvement. The Improvement Guide: a practical approach to enhancing organizational performance. 2nd ed. San Francisco, CA: Jossey-Bass; 2009. p. 89 - 108.

24. Hawe C, Ellis KS, Cairns CJ, Longmate A. Reduction of ventilator-associated pneumonia: Active versus passive guideline implementation. Intensive Care Medicine. 2009;35:1180-6.

25. Longmate AG, Ellis KS, Boyle L, Maher S, Cairns CJS, Lloyd SM, et al. Elimination of central-venous-catheterrelated bloodstream infections from the intensive care unit. BMJ Qual Saf 2011;20:174-80.

26. Dixon-Woods M, Bosk CL, Aveling EL, Goeschel CA, Pronovost PJ. Explaining Michigan: developing an ex post theory of a quality improvement program. Milbank Quarterly 2011;89:167-205.

27. Batalden PB, Davidoff F. What is "quality improvement" and how can it transform healthcare? BMJ Qual Saf 2007;16:2-3.

28. Langley GJ, Moen R, Nolan KM, Nolan TW, Norman CL, Provost LP. Developing Improvement Capability. The Improvement Guide: a practical approach to enhancing organizational performance 2nd ed. San Francisco, CA: Jossey-Bass; 2009. p. 331-47.

29. Davidoff F. Heterogeneity is not always noise: lessons from improvement. JAMA 2009;302:2580-6.

30. Ovretveit JC, Shekelle PG, Dy SM, McDonald KM, Hempel $S$, Pronovost $\mathrm{P}$, et al. How does context affect interventions to improve patient safety? An assessment of evidence from studies of five patient safety practices and proposals for research. BMJ Qual Saf 2011;20:604-10.

31. Stevens DP, Shojania KG. Tell me about the context, and more. BMJ Qual Saf 2011;20:557-9.

\section{Declaration of interests}

Andrew Longmate, Malcolm Daniel, and Shaun Maher completed Patient Safety Fellowships supported by the Scottish Patient Safety Programme. Malcolm Daniel was a Health Foundation quality improvement fellow (2010-2011). Louise Boyle is nursing lead for 


\section{BMJ Quality Improvement Reports}

the critical care work stream of the Scottish Patient Safety Programme at Stirling Royal Infirmary. An abstract of this work was presented in part at the $\mathrm{IHI}$ 22nd Annual National Forum on Quality Improvement in Healthcare (Orlando, FI, December 2010).

*Note the Stirling Royal Infirmary Intensive Care Unit, Livilands, Stirling FK8 2AU, UK closed in July 2011 when the clinical work, patients and teams was transferred to the new Forth Valley Royal Hospital, Stirling Road, Larbert, FK5 4WR.

\section{Acknowledgements}

We would like to thank the staff of both the ICUs and highlight the contributions of Heather Riddoch, Martyn Hawkins, Mike Williamson, Marianne Mallice, Pam Scott, Michelle Fraser, Lorna Hutchison and Janice Grant.

We thank Suzanne M Lloyd for help with statistical analysis. We would like to thank Jane Roessner, Frank Davidoff and Val Weber of the Institute for Healthcare Improvement for their help in reviewing earlier versions of the manuscript and providing suggestions on how to make it better. 